\title{
The effect of insulin on bone mineral density among women with type 2 diabetes: a SWAN Pharmacoepidemiology study
}

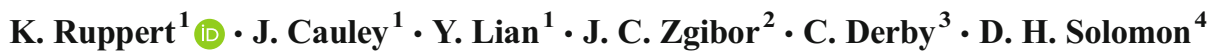

Received: 24 May 2017 / Accepted: 13 October 2017 /Published online: 27 October 2017

(C) The Author(s) 2017. This article is an open access publication

\begin{abstract}
Summary This was a longitudinal study examining the effects of insulin use on bone mineral density loss. Insulin use was found to be associated with greater bone mineral density loss at the femoral neck among women with diabetes mellitus.

Introduction Women with diabetes mellitus (DM) have higher bone mineral density (BMD) and experience slower BMD loss but have an increased risk of fracture. The data regarding the effect of insulin treatment on BMD remains conflicted. We examined the impact of insulin initiation on BMD.

Methods We investigated the annual changes in BMD associated with the new use of insulin among women with DM in the Study of Women's Health Across the Nation (SWAN). Propensity score (PS) matching, which is a statistical method that helps balance the baseline characteristics of women who did and did not initiate insulin, was used. Covariates with a potential impact on bone health were included in all models.
\end{abstract}

K. Ruppert

ruppertk@pitt.edu

1 Department of Epidemiology, Graduate School of Public Health, University of Pittsburgh, 4420 Bayard St Suite 600, Pittsburgh, PA 15260, USA

2 Department of Epidemiology and Biostatistics, University of South Florida, Tampa, FL, USA

3 Department of Neurology, Albert Einstein College of Medicine, New York, NY, USA

4 Division of Rheumatology, Division of Pharmacoepidemiology, Brigham and Women's Hospital, Harvard Medical School, Boston, MA, USA
Mixed model regression was used to test the change in BMD between the two groups. Median follow-up time was 5.4 years.

Results The cohort consisted of 110 women, mean age, 53.6 years; $49 \%$ white and $51 \%$ black. Women using insulin $(n=55)$ were similar on most relevant characteristics to the 55 not using insulin. Median diabetes duration for the user group was 10 vs. 5.0 years for the non-user group. There was a greater loss of BMD at the femoral neck among insulin users $(-1.1 \%)$ vs non-users $(-0.77 \%)(p=0.04)$. There were no differences in BMD loss at the spine $-0.30 \%$ vs $-0.32 \%$ $(p=0.85)$ or at the total hip $-0.31 \%$ vs $-0.25(p=0.71)$, respectively.

Conclusions Women with T2DM who initiated insulin experienced a more rapid BMD loss at the femoral neck as compared to women who did use insulin.

Keywords Bone mineral density · Diabetes mellitus . Fracture $\cdot$ Insulin $\cdot$ Women

\section{Introduction}

Diabetes mellitus (DM) and its complications contribute to significant morbidity and mortality. Not only has DM been shown to increase the risk for microvascular complications (retinopathy, nephropathy, neuropathy) and macrovascular complications (coronary heart disease, stroke, peripheral vascular disease), but it also may have affects on bone and fracture risk [1-5]. Many studies have shown that women with DM have higher bone mineral density (BMD) than women without DM. However, those with DM tend to have greater bone loss and a higher rate of fracture [6-19]. Most agree that endogenous insulin has an anabolic effect on bone [18-22]. 
However, there are several mechanisms by which diabetes can affect bone health. These include hyperglycemia, hyperinsulinemia and insulin-like growth factor-1 (IGF-1), antidiabetic agents, and diabetes disease severity to name a few [19-36].

Hyperglycemia effects bone health by increasing oxidative stress and elevating levels of advanced glycation end products (AGE) [19, 23, 25, 26, 31, 32, 36]. Hyperinsulinemia has been thought to increase BMD [18, 19, 21-27, 32] by some, whereas others have found it to decrease BMD [23, 28, 31, 36]. Srikanthan found that hyperinulinemia more negatively affects bone than hyperglycemia does.

The effects of medications used for T2DM likely play a role in bone health. Numerous studies have shown that thiazolidinediones are associated with decreases in BMD and increase in fracture rates [16, 23, 24, 32, 36]. In contrast, metformin, glucagon-like peptide-1 receptor agonists (GLP-1) and sulfonylureas have been reported to decrease the risk for fracture and BMD loss [23-26, 32, 36]. Studies looking at the effect of insulin in particular have yielded conflicting results. Three studies found that exogenous insulin increased BMD $[21,23,32]$, three studies found no effect on bone [29, 30, 36], and three studies found that insulin decreased bone loss or increased rate of fracture [24, 37, 38]. Barrett-Conner et al. found that exogenous insulin therapy removes the impact of endogenous insulin as an anabolic agent on bone.

Insulin is usually a last line of treatment for those with T2DM. Therefore, people who initiate insulin therapy are more likely have more comorbidities as well as a more severe disease progression. Several studies found that advanced disease also increased the risk for fracture [21, 24-26]. One study did not find any effect on fracture. [29].

At present, there is little known about the longitudinal effects of insulin initiation on bone health. The purpose of the current study was to evaluate the effect of insulin initiation on longitudinal changes in BMD, using the pharmacoepidemiology statistical new user design methodology [39] and propensity score matching.

\section{Methods}

\section{Study design}

The Study of Women's Health Across the Nation (SWAN) began in 1996-1997 to study health changes during mid-life in a multi-ethnic community-based cohort of 3302 women. The overall aim of SWAN is to examine a wide variety of health-related issues as women transition through menopause. The full study design and procedures, including recruitment and medication collection protocols have been described in detail elsewhere [37]. Briefly, seven sites across the USA enrolled women between the ages of 42 and 52 if they were pre or early perimenopausal, had an intact uterus, had at least one intact ovary, were not currently taking hormone therapy or oral contraceptives, were not pregnant or lactating, and had $\geq 1$ menstrual period in the previous 3 months. After enrollment, women were seen annually. They were instructed to bring in all medication containers, and trained interviewers transcribed all medications onto the study form. Five of the seven sites conducted a bone health study, with BMD as one of its main outcomes; this involved 2365 women.

The current analysis examined whether the initiation of insulin in subjects with DM was associated with an increased loss of BMD at the femoral neck (FN) and spine and if insulin increased the risk for fracture. To examine this, we compared the annualized percent rate of change in BMD among diabetic women who started insulin compared to non-users of insulin. The visit before insulin initiation was considered baseline for the user group. For those with DM who never reported insulin use, we randomly selected a frequency-matched visit to establish a comparable baseline. This was to ensure a similar distribution of the baseline visit between the two groups. Using propensity score matching, we created a matched cohort of insulin users and non-users. These analyses were carried out using propensity score-matched regression.

\section{Study sample}

Of the 2365 women in the bone cohort, 317 had DM based on a longitudinal review of the subject's history of [1] fasting baseline glucose level $\geq 126 \mathrm{mg} / \mathrm{dl}$ at two or more visits, [2] self-report of DM, or [3] hypoglycemic medications or insulin use. We excluded women from this analysis if they were using insulin at the time of enrollment into the SWAN study $(n=29)$. Those that had no history of insulin use were frequency matched with the insulin initiators based on the calendar date of the index visit. Those who could not be matched were excluded $(n=4)$. This left 284 women in the study. Once propensity score (PS) matching was conducted, the final analytic sample consisted of 55 subjects in each group (Fig. 1).

\section{Assessment of medication use}

Medication use was assessed at each SWAN study visit. Participants were asked about medications taken in the last 3 months, and responses were verified by visual inspection of medication bottles. If the participant forgot to bring medication containers to the study visit, a review of medication lists was performed. Each medication was then classified into its generic name and assigned a code according to a computerized medication dictionary (Iowa Drug Information Service (IDIS) Drug Vocabulary, College of Pharmacy, University of Iowa, Iowa City, IA). Dosage information was not consistently listed and thus was not used for these analyses. Further, all 


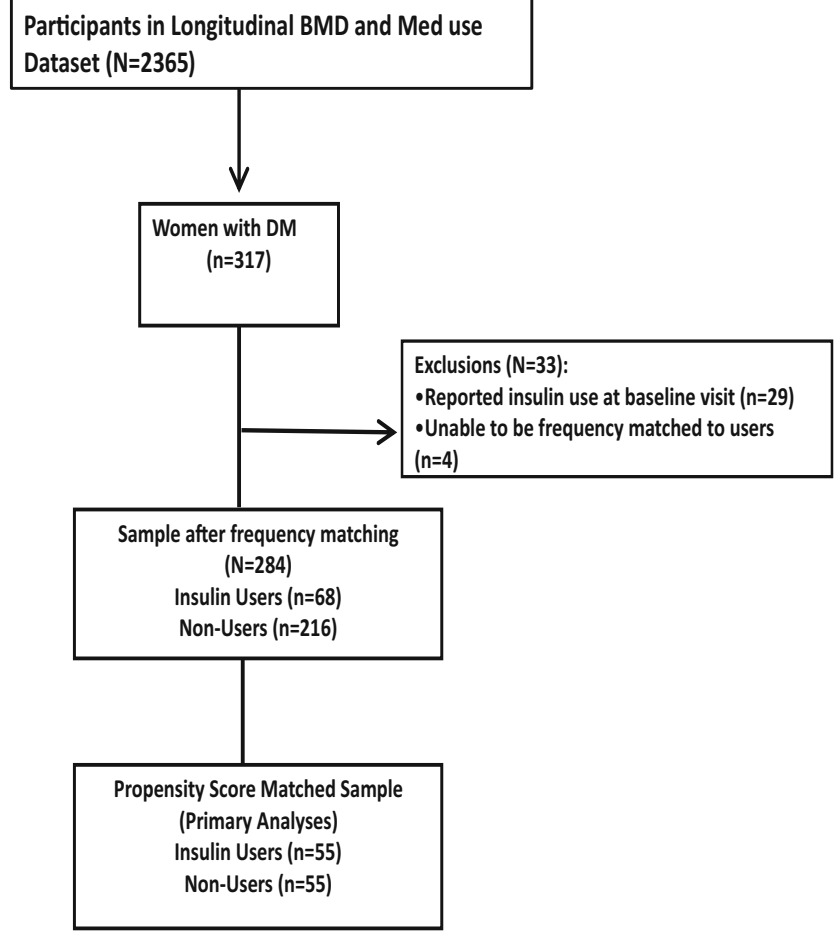

Fig. 1 Flow chart of subject inclusion. This figure demonstrates the assembly of the primary analytic cohort. The primary cohort used propensity scores to match initiators of insulin with those who never used insulin

insulin preparations were considered equal for the purposes of these analyses.

\section{Bone mineral density measurements}

The BMD $\left(\mathrm{g} / \mathrm{cm}^{2}\right)$ of the lumbar spine and femoral neck were measured annually using Hologic instruments (Hologic Inc., Waltham, Massachusetts). Three sites used Hologic 4500A models at baseline; two of these sites later upgraded to Discovery models, one at follow-up visit 12 and one at follow-up visit 13. Two sites started with 2000 models at baseline and both of upgraded to 4500 models at follow-up visit 8 . Each site that upgraded its hardware scanned 40 volunteers on both old and new machines to develop crosscalibration regression equations, which were applied by the SWAN Coordinating Center. A standard quality control (QC) program was conducted in collaboration with QC centers at Synarc Inc. (San Francisco, CA) from baseline to follow-up visit 10 and with the USCF DXA Quality Assurance Center (San Francisco, CA) thereafter. QC included daily phantom measurements, quarterly review of the daily QC plots by the QC centers with correction factors applied for drift if needed, local site review of all scans, and review of problem scans by a member of the SWAN Bone Committee. Short-term in vivo measurement variability was $0.014 \mathrm{~g} / \mathrm{cm}^{2}(1.4 \%)$ for the LS and $0.016 \mathrm{~g} / \mathrm{cm}^{2}(2.2 \%)$ for the FN.

\section{Statistical analysis}

After comparing the baseline characteristics between the user and non-user groups, it was found that there was a considerable imbalance between the two. Therefore, we chose to improve the comparability of groups by using propensity score (PS) as a method for matching women in the user group to women in the non-user group. PS matching usually involves the formation of pairs of treated and untreated subjects with similar propensity scores. Greedy matching is commonly used for the formation of these pairs. This means that for a given treated subject, the closest untreated subject within the specified caliper distance is selected for matching to the treated subject. Logistic regression models are mostly used and those baseline variables that influence treatment assignment are included in the model. In this analysis, the following variables were used to calculate the PS: age, race, BMI, smoking status, menopausal status, baseline FN and spine BMD, and number of comorbid conditions (anemia, hypertension, hyperlipidemia, migraine, stroke, osteoarthritis, myocardial infarction, angina, osteoporosis, thyroid disease, any cancer). A greedy matching caliper was set at 0.2 of the standard deviation of the logit of the PS [40].

Descriptive statistics (mean, median, and range) of the baseline demographic variables were calculated. Continuous variables were analyzed using Student's $t$ test, or the nonparametric analog; whereas categorical variables were analyzed using $\chi^{2}$. Variables were transformed where necessary. Standardized mean differences (SMD) were then calculated to examine improvement in balance between the two groups.

To compare the annualized rate of change in BMD among the insulin users and non-users, a mixed-effects regression modeling strategy, allowing for a random intercept and slope, was used [41]. Factors selected a priori for inclusion in the base models included years from the baseline visit as a continuous linear covariate and several covariates known to be possible correlates of BMD. Study site, race (Caucasian, African American, Chinese, Japanese), and diabetes duration were time-invariant, whereas age, BMI, medications known to affect BMD (hormone use, thiazide diuretics, bisphosphonates), number of comorbid conditions, fasting blood glucose (FBG), calcium, vitamin D, physical activity, and menopausal status were time varying. Since some antidiabetic medications have been reported to affect BMD, concurrent use of metformin, sulfonylureas, and thiazolidinediones were also included. A sensitivity analysis removing those that used thiazide diuretics at baseline was also conducted. For consistency, if a covariate was found to be significant at one anatomical site (i.e., femoral neck), that covariate was forced into the other site. Therefore, all final models for each comparison group contain the same covariates and only included the a priori variables and other covariates with $P$ values $<0.05$. SAS version 9.3 (SAS Institute, Inc., Cary, NC) was used for the analyses. 
A secondary analysis of BMD change over time in only the insulin user group was carried out using a piecewise mixed model regression. Again a random intercept and slope was use. Since it was thought that insulin might have a cumulative effect on bone, a longer follow-up period was examined after initiation. Therefore, time in the model included up to 3 years prior to insulin initiation and all data points after.

\section{Results}

The analytic sample consisted of 110 women; $49 \%$ were white and $51 \%$ black. After PS matching, women using insulin $(n=55)$ were similar in age, BMI, FN BMD, educational attainment, smoking status, menopausal status, calcium, vitamin D, thiazide diuretic, and bisphosphonate use (Table 1) compared to the women not using insulin $(n=55)$. Insulin users had a higher FBG (187.8 mg/dl vs $144.3 \mathrm{mg} / \mathrm{dl})$ and were concurrently on thiazolidinediones, metformin, and sulfonylureas compared to the non-user group. Diabetes duration for the user group was 9.1 vs. 5.7 years for the non-user group. The mean number of BMD scans was 4.0 (STD = 2.9) per person.

Figure 2 shows the annual percent change in BMD at the FN spine and total hip for the PS matched sample. After adjusting for age, BMI, race, site, menopausal status, FBG, smoking status, diabetes duration, calcium, vitamin D, hormone, bisphosphonate, thiazide diuretic, metformin, sulfonylurea, physical activity, and thiazolidinedione use, those using insulin had a greater decrease in BMD at the FN ( -1.1 vs $-0.77 \% ; p=0.04$ ) than those not using insulin. The annual change at the spine and total hip was similar between the two groups. Since thiazide diuretic use has been shown to increase BMD, a sensitivity analysis was conducted removing women who were using these at baseline (Fig. 3). The results were similar to the primary analysis, with users losing $1.23 \%$ at the FN compared to $-0.87 \%$ in the non-users $(p=0.046)$. Again, there was no difference at the spine or total hip between the two groups.

Figure 4 illustrates the results from the secondary piecewise mixed model analysis for those who initiated insulin. After adjusting for age, race, BMI, menopausal status, and physical activity the within women slope after insulin initiation at both the FN and the total hip are significant (FN $-1.3 \% ; p=0.001$, total hip $-0.75 \% p=0.005)$. The comparison of the slopes pre- and post-insulin use at all three anatomic sites were not significant. As an exploratory analysis, we examined the effect of insulin on fractures. There were a total of 14 fractures among women in the analysis sample. The most common fracture was foot $(n=6)$, followed by arm above the wrist $(n=2)$, leg $(n=2)$, ankle $(n=2)$, patella $(n=1)$, and other $(n=1)$ fracture. Nine of the 14 fractures were non-traumatic. After adjusting for age, race, BMI, baseline FN BMD, and menopausal status, there was no difference in fracture risk (HR $1.4 ; 95 \%$ CI $0.43-4.3 ; P$ value $=0.81$.

\section{Discussion}

We studied a longitudinal cohort of 110 women with diabetes who were enrolled in SWAN to determine if there was a difference in BMD loss and fracture risk between those that initiated insulin and those who did not. Initiation of insulin was found to be associated with greater rates of decline at the femoral neck after adjusting for age, BMI, race, site, menopausal status, current smoking status, FBG, education, diabetes duration, hormone user, bisphosphonate, calcium, vitamin D, physical activity, thiazide diuretic, metformin, sulfonylurea, and thiazolidinedione use. There were no differences found in BMD loss at the spine or total hip.

There are several mechanisms that can affect bone health in T2DM. First, hyperglycemia has been found to attenuate bone density by reducing osteoblast function and number, inhibiting osteoblast maturation and bone mineralization, impairing the response to 1, 25 hydroxy vitamin D3, and inducing glycation of various proteins and produce advanced glycosylation end products (AGEs) [19, 23, 25, 26, 31, 32, 36]. In cortical bone, an accumulation of AGEs can affect the rigidity of the bone but does not seem to affect bone mineralization [23]. The result seen in the FN of this study may suggest that exogenous insulin use may be affecting bone loss independent of FBG levels which were consistently higher in those that initiated insulin.

Second, insulin has both a direct and indirect effect on osteogenesis. The direct effect is mediated through insulin and insulin-like growth factor-1 (IGF-1) receptors located on the osteoblast [23]. The indirect effect of insulin is mediated through the control of blood glucose levels and its effects on the parathyroid hormone, IGF-1, and vitamin D. In animal and clinical studies, it has been repeatedly shown that those with type $1 \mathrm{DM}$ (T1DM) the BMD of the FN is lower than those with T2DM. This is thought to be due to a lack of insulin in T1DM but that in T2DM it could be explained by a higher BMI [18, 19, 23, 25, 26]. Since it is known that exogenous insulin increases weight gain, in this study, both the insulin and non-insulin user groups were matched on BMI at baseline. Also, an analysis was carried out to see if there was a significant change in BMI between the two groups. We found a small but not statistically significant difference in BMI of 0.38 (insulin starters) vs -0.75 (non-starters) $(p=0.25)$. Also, BMI which was incorporated in the models as a time varying covariate was not found to be significant at any site.

Third, other antidiabetic agents have been found to have both positive and negative impacts on bone health. 
Table 1 Baseline characteristics of women with type 2 diabetes mellitus

\begin{tabular}{|c|c|c|c|c|c|c|c|}
\hline \multirow[b]{2}{*}{ Variable } & \multicolumn{2}{|c|}{ Total $N=110$} & \multicolumn{2}{|c|}{ Non-insulin user $N=55$} & \multicolumn{2}{|c|}{ Insulin user $N=55$} & \multirow[t]{2}{*}{ SMD } \\
\hline & $n$ & $\%$ & $n$ & $\%$ & $n$ & $\%$ & \\
\hline Age, mean (SD) & 53.6 & 5.30 & 53.9 & 5.70 & 53.3 & 4.90 & 0.10 \\
\hline BMI, mean (SD) & 35.7 & 6.70 & 35.5 & 6.50 & 35.8 & 7.00 & 0.05 \\
\hline Diabetes duration, mean (SD) & 7.4 & 4.80 & 5.7 & 5.00 & 9.1 & 4.00 & 0.76 \\
\hline Femoral neck BMD, mean (SD) & 0.92 & 0.15 & 0.91 & 0.15 & 0.93 & 0.14 & 0.16 \\
\hline Spine BMD, mean (SD), & 1.2 & 0.16 & 1.1 & 0.16 & 1.2 & 0.16 & 0.27 \\
\hline Total hip BMD, mean (SD) & 1.07 & 0.16 & 1.05 & 0.16 & 1.09 & 0.16 & 0.23 \\
\hline Total comorbidities, mean (SD) & 2.6 & 1.20 & 2.5 & 1.30 & 2.8 & 1.10 & 0.22 \\
\hline Follow-up (years) mean (SD) & 6.2 & 4.60 & 5.7 & 5.00 & 6.8 & 4.10 & 0.25 \\
\hline Fasting blood glucose (mg/dl) & 166.3 & 73.70 & 144.3 & 61.20 & 187.8 & 79.00 & 0.62 \\
\hline Physical activity & 6.8 & 1.7 & 7.0 & 1.8 & 6.6 & 1.7 & 0.25 \\
\hline \multicolumn{8}{|l|}{ Educational attainment } \\
\hline Up to high school & 47 & 0.43 & 25 & 0.45 & 22 & 0.40 & \multirow[t]{2}{*}{0.11} \\
\hline Beyond high school & 63 & 0.57 & 30 & 0.55 & 33 & 0.60 & \\
\hline \multicolumn{8}{|l|}{ Tobacco use } \\
\hline Current & 33 & 0.30 & 18 & 0.33 & 15 & 0.27 & 0.12 \\
\hline \multicolumn{8}{|l|}{ Site } \\
\hline Michigan & 49 & 0.45 & 25 & 0.45 & 24 & 0.44 & 0.04 \\
\hline Mass General & 21 & 0.19 & 11 & 0.20 & 10 & 0.18 & 0.05 \\
\hline UC Davis & 7 & 0.06 & 2 & 0.04 & 5 & 0.09 & 0.23 \\
\hline UCLA & 7 & 0.06 & 3 & 0.05 & 4 & 0.07 & 0.08 \\
\hline Pittsburgh & 26 & 0.24 & 14 & 0.25 & 12 & 0.22 & 0.09 \\
\hline \multicolumn{8}{|l|}{ Race } \\
\hline White & 54 & 0.49 & 28 & 0.51 & 26 & 0.47 & \multirow[t]{2}{*}{0.07} \\
\hline Black & 56 & 0.51 & 27 & 0.49 & 29 & 0.53 & \\
\hline \multicolumn{8}{|l|}{ Menstrual status } \\
\hline Pre & 8 & 0.07 & 5 & 0.09 & 3 & 0.05 & 0.14 \\
\hline Early/late peri & 26 & 0.24 & 13 & 0.24 & 13 & 0.24 & 0.00 \\
\hline Post & 63 & 0.57 & 32 & 0.58 & 31 & 0.56 & 0.04 \\
\hline Unknown* & 13 & 0.12 & 5 & 0.09 & 8 & 0.15 & 0.17 \\
\hline \multicolumn{8}{|l|}{ Medication use } \\
\hline Hormone replacement & 11 & 0.10 & 4 & 0.07 & 7 & 0.13 & 0.18 \\
\hline Bisphosphonate & 2 & 0.02 & 1 & 0.02 & 1 & 0.02 & 0.00 \\
\hline Thiazide diuretic & 27 & 0.25 & 14 & 0.25 & 13 & 0.24 & 0.04 \\
\hline Vitamin D & 16 & 0.15 & 10 & 0.18 & 6 & 0.11 & 0.21 \\
\hline Calcium & 17 & 0.15 & 8 & 0.15 & 9 & 0.16 & 0.05 \\
\hline \multicolumn{8}{|l|}{ Oral hypoglycemics } \\
\hline Thiazolidinedione & 16 & 0.15 & 3 & 0.05 & 13 & 0.24 & 0.53 \\
\hline Metformin & 66 & 0.60 & 31 & 0.56 & 35 & 0.64 & 0.15 \\
\hline Sulfonlyurea & 38 & 0.35 & 7 & 0.13 & 31 & 0.56 & 1.00 \\
\hline
\end{tabular}

SMD standardized mean difference

*Unknown because of hormone therapy or surgery
For example, studies have found that metformin and sulfonylureas have positive effects on bone health. Whereas, thiazolidinediones have a negative impact. Exogenous insulin has remained controversial. While numerous studies have found endogenous insulin to have an anabolic effect on bone
[18-22], Dutta found that exogenous insulin therapy removes the impact of endogenous insulin. He also found that insulin will inhibit C-peptide release which leads to a negative impact on bone [37]. Newer hypoglycemic agents such as glucagontype peptide (GLP-1) agonists have been found to be 


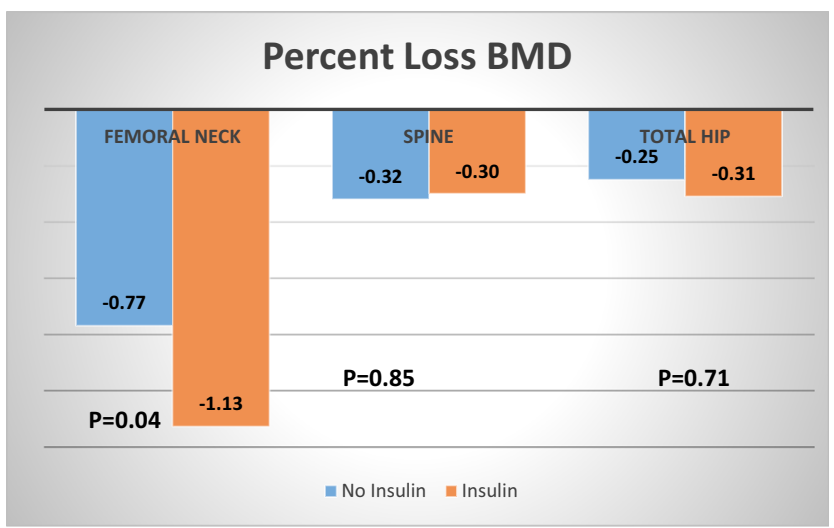

Fig. 2 Annualized percent of bone mineral density loss between insulin users and non-users. All BMD models adjusted for age, BMI, race, site, menopausal status, current smoking status, FBG, physical activity, education, diabetes duration, hormone user, bisphosphonate, calcium, vitamin $\mathrm{D}$, thiazide diuretic, metformin, sulfonylurea, and thiazolidinedione use

associated with both bone loss and gain and sodium-glucose co-transporter 2 (SGLT2) inhibitors where found to be detrimental to bone. Since these latter drugs were not introduced in the USA at the time of data collection, we were unable to ascertain the effects of these drugs on this study.

Disease severity, treatment modalities, and duration of diabetes have been found to increase the risk for both microvascular and macrovascular condition [1-5] as well as increase the risk for fracture. In this study, those that initiated insulin had a higher number of comorbid conditions (2.8 vs 2.5), higher FBG (187.8 vs $144.3 \mathrm{mg} / \mathrm{dl})$ and had T2DM for a longer period of time (9.1 vs 5.1 years) all indicating an advanced disease state. To control for these potential confounding covariates, we PS matched or adjusted for these covariates in all models.

Four prior studies examined insulin use and its effects on BMD. The current results showing an association between

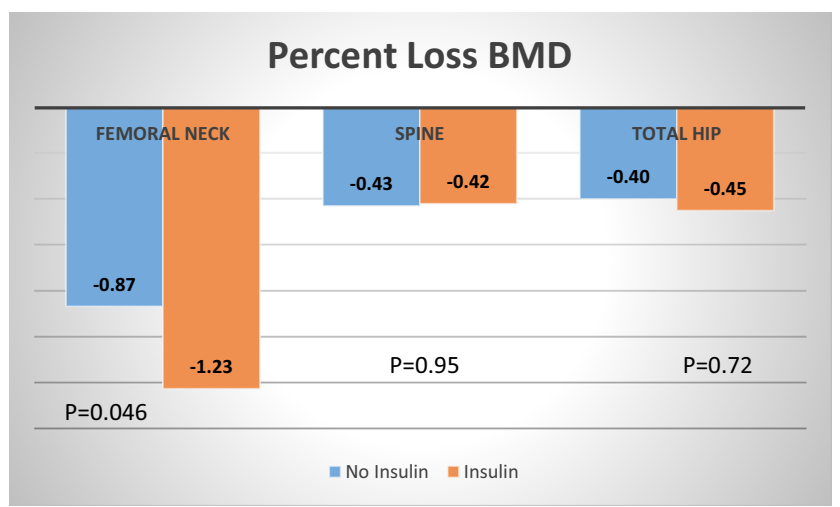

Fig. 3 Annual Percent of bone mineral density loss between insulin users and non-users after removing thiazide diuretic users at baseline. All BMD models adjusted for age, BMI, race, site, menopausal status, current smoking status, FBG, education, physical activity, diabetes duration, hormone user, bisphosphonate, calcium, vitamin $\mathrm{D}$, thiazide diuretic, metformin, sulfonylurea, and thiazolidinedione use

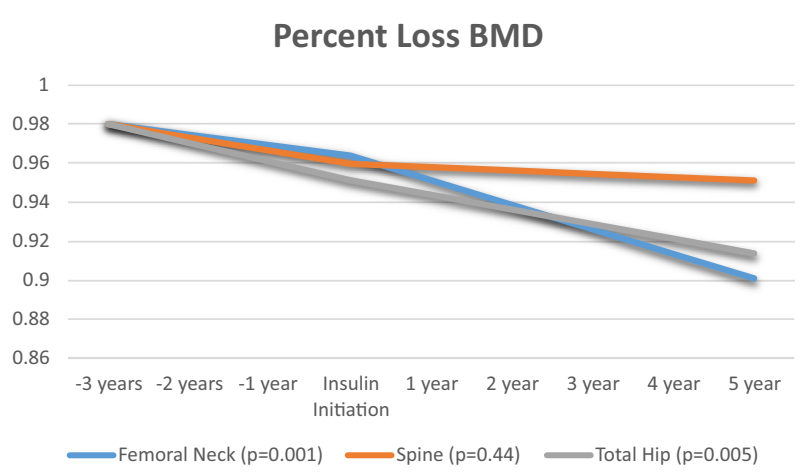

Fig. 4 Annual percent of bone mineral density loss before and after insulin initiation. Models adjusted for age, race, BMI, menopausal status, and physical activity $P$ values reflect within person change after insulin initiation

insulin use and a decrease in BMD at the $\mathrm{FN}$ are consistent with findings from a prior study by Dutta [37]. While both studies included women of similar age (53 years old), the SWAN database allowed us to include additional covariates known to affect BMD, such as menopausal status, coexisting comorbid conditions, and medications that affect BMD such as hormone therapy, thiazide diuretics, and bisphosphonates, and socio-economic factors. Further, our study had a median follow-up of 5.4 years, compared to only 1 year, which further distinguishes the results of the current analysis.

Our study has several strengths, including a well characterized cohort and a longitudinal design allowing up to 13 data points per person. Also, we could track BMD loss from the onset of insulin therapy in conjunction with other medications for diabetes and medications for other conditions while adjusting for menopausal status. The relationship between menopausal status and BMD has been well characterized in SWAN [42], adding to the robust nature of the current analyses. Compared to the rates of BMD loss in a previously published SWAN study whose mean BMI was 27, our rates of bone loss are similar. Since BMI has a protective effect on bone, we would have expected a much slower rate of decline in this study. Thus, it supports the conclusion that insulin use may be a major contributing factor for the accelerated bone loss. Most prior studies have been cross-sectional, have not adjusted for menopausal status or other bone active medications, comorbid conditions, or did not employ a new user design. New user designs are the preferred method in pharmacoepidemiology studies [39]. Though a new user design does not create balanced baseline comparisons, we were able to achieve this using propensity score matching.

There are, however, limitations to the current study. As this is an observational analysis, imbalances in subject characteristics could explain differences in BMD; thus, determining causation would require a RCT. Further, to balance our groups, we sacrificed statistical power for an improvement in the balance of subject characteristics. In 
this study, power ranged from 85 to $98 \%$ with an alpha of 0.05 for this analysis. Finding a significant results indicates sufficient power. However, whenever a statistical analysis is performed and results interpreted, there is always a finite chance that the results are purely by chance. This is an inherent limitation of any statistical analysis and cannot be done away with. Another limitation could have been that some women had type $1 \mathrm{DM}$. In an attempt to eliminate this possibility, those women that were on insulin at SWAN baseline were removed from the study cohort $(n=29)$. Finally, since SWAN was specifically designed for assessing women as they transition through menopause, these results may not be generalizable to men. Despite these limitations, to our knowledge, this is the first longitudinal study to examine insulin use and bone loss that was adjusted for potential confounders such as comorbid conditions, socioeconomic status, and menopausal status while using the preferred pharmacoepidemiologic statistical new user design methodology.

In conclusion, we found that insulin use was associated with increased bone loss at the FN but not at the spine or total hip. These results suggest that when making treatment decisions for women with diabetes special consideration should be given to women who are at a high risk for osteoporosis.

Acknowledgments We thank the study staff at each site and all the women who participated in SWAN.

Funding information The Study of Women's Health Across the Nation (SWAN) has grant support from the National Institutes of Health (NIH), DHHS, through the National Institute on Aging (NIA), the National Institute of Nursing Research (NINR), and the NIH Office of Research on Women's Health (ORWH) (Grants U01NR004061; U01AG012505, U01AG012535, U01AG012531, U01AG012539, U01AG012546, U01AG012553, U01AG012554, U01AG012495). The content of this manuscript is solely the responsibility of the authors and does not necessarily represent the official views of the NIA, NINR, ORWH, or the NIH. Clinical centers: University of Michigan, Ann Arbor-Siobán Harlow, PI 2011-present, MaryFran Sowers, PI 1994-2011; Massachusetts General Hospital, Boston, MA — Joel Finkelstein, PI 1999-present, Robert Neer, PI 1994-1999; Rush University, Rush University Medical Center, Chicago, IL-Howard Kravitz, PI 2009-present, Lynda Powell, PI 1994-2009; University of California, Davis/Kaiser-Ellen Gold, PI; University of California, Los Angeles-Gail Greendale, PI; Albert Einstein College of Medicine, Bronx, NY - Carol Derby, PI 2011-present, Rachel Wildman, PI 2010-2011; Nanette Santoro, PI 2004-2010; University of Medicine and Dentistry-New Jersey Medical School, Newark-Gerson Weiss, PI 1994-2004; and the University of Pittsburgh, Pittsburgh, PA - Karen Matthews, PI.

NIH program office: National Institute on Aging, Bethesda, MDChhanda Dutta 2016-present, Winifred Rossi 2012-2016, Sherry Sherman 1994-2012, Marcia Ory 1994-2001; National Institute of Nursing Research, Bethesda, MD - Program officers.

Central laboratory: University of Michigan, Ann Arbor-Daniel McConnell (Central Ligand Assay Satellite Services).

Coordinating center: University of Pittsburgh, Pittsburgh, PA-Maria Mori Brooks, PI 2012-present; Kim Sutton-Tyrrell, PI 2001-2012; New England Research Institutes, Watertown, MA-Sonja McKinlay, PI 1995-2001.
Steering committee: Susan Johnson, Current Chair; Chris Gallagher, and Former Chair.

\section{Compliance with ethical standards}

Conflicts of interest Dan H. Solomon receives salary support from institutional research grants from Eli Lilly, Amgen, Pfizer, AstraZeneca, Genentech, and Corrona. He also receives royalties from UpToDate and serves in unpaid roles in studies funded by Pfizer and Eli Lilly. Kristine Ruppert, Jane Cauley, Yijuan Lian, Carol Derby, and Janice Zgibor declare they have no conflict of interest.

Open Access This article is distributed under the terms of the Creative Commons Attribution-NonCommercial 4.0 International License (http:// creativecommons.org/licenses/by-nc/4.0/), which permits any noncommercial use, distribution, and reproduction in any medium, provided you give appropriate credit to the original author(s) and the source, provide a link to the Creative Commons license, and indicate if changes were made.

\section{References}

1. Fong DS, Aiello LP, Ferris FL 3rd, Klein R (2004) Diabetic retinopathy. Diabetes Care 27:2540-2553

2. The DCCT Research Group (1993) The effect of intensive treatment of diabetes on the development and progression of long-term complications in insulin-dependent diabetes mellitus. N Engl J Med 329:977-986

3. UK Prospective Diabetes Study Group (1998) Intensive blood glucose control with sulphonylureas or insulin compared with conventional treatment and risk of complications in patients with type 2 diabetes (UKPDS 33). Lancet 352:837-853

4. Keenan HA, Costacou T, Sun JK, Doria A, Cavellerano J, Coney J, Orchard TJ, Aiello LP, King GL (2007) Clinical factors associated with resistance to microvascular complications in diabetic patients of extreme disease duration: the 50-year medalist study. Diabetes Care 30:1995-1997

5. Gross JL, de Azevedo MJ, Silveiro SP, Canani LH, Caramori ML, Zelmanovitz T (2005) Diabetic nephropathy: diagnosis, prevention, and treatment. Diabetes Care 28:164-176

6. Khalil N, Sutton-Tyrrell K, Strotmeyer ES, Greendale GA, Vuga M, Selzer F, Crandall CJ, Cauley JA (2011) Menopausal bone changes and incident fractures in diabetic women: a cohort study. Osteoporos Int 22(5):1367-1376

7. Krakauer JC, McKenna MJ, Buderer NF, Rao DS, Whitehouse FW, Parfitt AM (1995) Bone loss and bone turnover in diabetes. Diabetes 44:775-782

8. Rosato MT, Schneider SH, Shapses SA (1998) Bone turnover and insulin-like growth factor I levels increase after improved glycemic control in non-insulin dependent diabetes mellitus. Calcif Tissue Int 63:107-111

9. Akin O, Göl K, Aktürk M, Erkaya S (2003) Evaluation of bone turnover in postmenopausal patients with type 2 diabetes mellitus using biochemical markers and bone mineral density measurements. Gynecol Endocrinol 17(1):19-29

10. Mansoor Karimifar, Morteza Aghajanpoor, Amirhossein Salari, Ahmadreza Zamani, Mansour Salesi, Peyman Motaghi (2012) Evaluation of bone loss in diabetic postmenopausal women. J Res Med Sci, Vol 17, No 11

11. Zakeri Z, Azizi Z, Mehrabifar H, Hashemi M (2011) Evaluation of bone mineral density in premenopausal women with type-2 diabetes mellitus in Zahedan, southeast Iran. JPMA 61:443 
12. Touminen, J. Mpolsc, P. Ronnemaa, T. (1999) Bone mineral density in patients with type 1 and type 2 diabetes. Diabetes care, Vol 22, Number 7

13. Strotmeyer ES, Cauley JA (2007) Diabetes mellitus, bone mineral density, and fracture risk. Curr Opin Endocrinol Diabetes Obes. 14(6):429-435

14. Khazai NB, Beck GR Jr, Umpierrez GE (2009) Diabetes and fractures: an overshadowed association. Curr Opin Endocrinol Diabetes Obes 16(6):435-445

15. Hans D, Duboeuf F, Schott AM, Horn S, Avioli LV, Drezner MK, Meunier PJ (1997) Effects of a new positioner on the precision of hip bone mineral density measurements. J Bone Miner Res 12: 1289-1294

16. Schwartz AV, Ewing SK, Porzig AM, McCulloch CE, Resnick HE, Hillier TA, Sellmeyer DE (2013) Diabetes and change in bone mineral density at the hip, calcaneus, spine, and radius in older women. Front Endocrinol 4:62

17. de Liefde II, van der Klift M, de Laet CEDH et al (2005) Bone mineral density and fracture risk in type- 2 diabetes mellitus: the Rotterdam study. Osteoporos Int 16:1713

18. Ma L, Oei L, Jiang L, Estrada K, Chen H, Wang Z et al (2012) Association between bone mineral density and type 2 diabetes mellitus: a meta-analysis of observational studies. Eur J Epidemiol 27(5):319-332

19. Vestergaard P, Rejnmark L, Mosekilde L (2007) Discrepancies in bone mineral density and fracture risk in patients with type 1 and type 2 diabetes- a meta-analysis. Osteoporos Int 18:427

20. Klein GL (2014) Insulin and bone: recent developments. World J Diabetes 5(1):14-16

21. Thrailkill KM, Lumpkin CK, Bunn RC, Kemp SF, Fowlkes JL (2005) Is insulin an anabolic agent in bone? Dissecting the diabetic bone for clues. Am J Physiol Endocrinol Metab 289(5):E735-E745

22. McCarty MF (1995) Anabolic effects of insulin on bone suggest a role for chromium picolinate in preservation of bone density. Med Hypotheses 45(3)

23. Ghodsi M, Larijani B, Ali Keshtkar A, Nasli-Esfahani E, Alatab S, Reza Mohajeri-Tehrani M (2016) Mechanisms involved in altered bone metabolism in diabetes: a narrative review. J Diabetes Metab Disord 15:52

24. Napoli N, Chandran M, Pierroz DD, Abrahamsen B, Schwartz AV, Ferrari SL (2017) Mechanisms of diabetes mellitus-induced bone fragility. Nat Rev Endocrinol 13:208-219

25. Sundararaghavan V, Mazur MM, Evans B, Liu J, Ebraheim NA (2017) Diabetes and bone health: latest evidence and clinical implications. Ther Adv Musculoskelet Dis 9(3):67-74

26. Hamann C, Kirschner S, Gunther K, Hofbauer L (2012) Bone, sweet bone-osteoporotic fractures in diabetes mellitus. Nat Rev Endocrinol 8:297-305

27. Barrett-Connor E, Kritz-Silverstein D (1996) Does hyperinsulinemia preserve bone? Diabetes Care 19:1388-1392
28. Ishii S, Cauley JA, Crandall CJ et al (2012) Diabetes and femoral neck strength: findings from the hip strength across the menopausal transition study. J Clin Endocrinol Metab 97(1):190-197

29. De Waard EAC, Koster A, Melai T, van Geel TA, Henry RMA, Schram MT, van den Bergh JPW (2016) The association between glucose metabolism status, diabetes severity and a history of fractures and recent falls in participants of 50 years and older - the Maastricht Study. Osteoporos Int 27(11):3207-3216

30. Starup-Linde J, Gregersen S, Vestergaard P (2016) Associations with fracture in patients with diabetes: a nested case-control study. BMJ Open

31. Srikanthan P, Crandall CJ, Miller-Martinez D, Seeman TE, Greendale GA, Binkley N, Karlamangla AS (2014) Insulin resistance and bone strength. Findings from the study of midlife in the United States. J Bone Miner Res Off J Am Soc Bone Miner Res 29(4):796-803

32. Yan W, Li X (2013) Impact of diabetes and its treatments on skeletal diseases. Front Med 7:81

33. Majumdar SR, Josse RG, Lin M, Eurich DT (2016) Does sitagliptin affect the rate of osteoporotic fractures in type 2 diabetes? Population-based cohort study. J Clin Endocrinol Metab 101(5): 1963-1969

34. Vestergaard P, Rejnmark L, Mosekilde L (2005) Relative fracture risk in patients with diabetes mellitus and the impact of insulin and oral antidiabetic medication on relative fracture risk. Diabetologia 48:1292

35. Kumar BS, Ravisankar A, Mohan A, Kumar DP, Katyarmal DT, Sachan A, Sarma KVS (2015) Effect of oral hypoglycaemic agents on bone metabolism in patients with type 2 diabetes mellitus and occurrence of osteoporosis. Indian J Med Res 141(4):431-437

36. Adami S (2009) Bone health in diabetes: considerations for clinical management. Curr Med Res Opin 25(5):1057-1072

37. Dutta M, Pakhetra R, Garg M (2012) Evaluation of bone mineral density in type 2 diabetes mellitus patients before and after treatment. Med J Armed Forces India 68(1):48-52

38. Wallander M, Axelsson KF, Nilsson AG, Lundh D, Lorentzon M (2017) Type 2 diabetes and risk of hip fractures and non-skeletal fall injuries in the elderly: a study from the fractures and fall injuries in the elderly cohort (FRAILCO). J Bone Miner Res 32:449-460. https://doi.org/10.1002/jbmr.3002

39. Ray WA (2003) Evaluating medication effects outside of clinical trials: new-user designs. Am J Epidemiol 158:915-920

40. Faries D, Leon A, Haro JM, Obenchain RL (2010) Analysis of Observational Health Care Data using SAS. SAS Institute

41. Brown H, Prescott R (2006) Applied mixed models in medicine, 2nd edn. Hoboken, NJ, John Wiley, Sons, Ltd

42. Greendale GA, Sowers M et al (2012) Bone mineral density loss in relation to the final menstrual period in a multi-ethnic cohort: results from the study of women's health across the nation (SWAN). J Bone Miner Res Off J Am Soc Bone Miner Res 27(1):111-118 